\title{
DINÂMICA SAZONAL DE NUTRIENTES EM ESTUÁRIO AMAZÔNICO
}

\author{
Seasonal dynamics of nutrients in an Amazon estuary
}

\author{
Sury de Moura Monteiro* \\ Maâmar El-Robrini ** \\ Igor Charles Castor Alves ***
}

\begin{abstract}
Resumo
Este artigo mostra a distribuição dos nutrientes no estuário do rio Paracauari, durante um ciclo hidrológico amazônico (2008), e no final do período de La Niña (abril de 2008). Esse estuário é influenciado por clima tropical úmido e meso-marés $(3 \mathrm{a} 4 \mathrm{~m})$, semi-diurna. A amostragem foi realizada em 10 estações em três períodos sazonais distintos: chuvoso (março), intermediário (junho) e menos chuvoso (setembro). Medimos "in situ" os parâmetros físico-químicos utilizando uma sonda multiparâmetro; analisamos os nutrientes dissolvidos (nitrato, nitrito, n-amoniacal, fosfato e silicato) por espectofotometria e o material particulado em suspensão por gravimetria. Observamos amplas variações sazonais nas concentrações dos parâmetros estudados. A temperatura da água (média de $28,58^{\circ} \mathrm{C}$ ) é bastante homogênea, típica das águas tropicais. $\mathrm{O}$ $\mathrm{pH}$ variou de ácido $(5,80)$ à alcalino $(7,86)$ e a salinidade entre 0,06 a 7,56 ambos com valores máximos na foz, devido à maior influência marinha. As águas são mal oxigenadas no período chuvoso (2,35 mg.L1) e bem no menos chuvoso (6,55 mg.L-1). As concentrações de material particulado em suspensão e de nutrientes foram máximas no período chuvoso devido ao aporte natural proveniente das áreas adjacentes.
\end{abstract}

Palavras-chave: Estuário amazônico;Rio Paracauari; Meso maré; Ilha do Marajó.

\begin{abstract}
This paper shows the nutrients distribution in the Paracauari estuary over a Amazon hydrological cycle (2008), and at the end of the period of La Niña (April 2008). This estuary is influenced by the humid tropical climate and meso-tidal ( 3 to $4 \mathrm{~m}$ ), semi-diurnal. Sampling was conducted at 10 stations in three distinct seasons: rainy (march), intermediate (june) and less rainy season (september). Were measured in situ physical and chemical parameters using a multiparameter probe; analyzed the dissolved nutrients (nitrate, nitrite, ammonia-N, phosphate and silicate) by spectrophotometry and particulate matter suspension, gravimetrically. We observed large seasonal variations in the concentrations of the studied parameters. The water temperature (average of $28.58^{\circ} \mathrm{C}$ ) is quite homogeneous, typical of tropical waters. The $\mathrm{pH}$ ranged acid (5.80) to alkaline (7.86) and salinity between 0.06 to 7.56 , with both maximum values at the mouth because of the greater marine influence. The waters are poorly oxygenated during the rainy season $(2.35 \mathrm{mg}$.L-1) and at the very least rainy (6.55 mg.L-1). The concentrations of the suspended particulate matter and nutrients reached a maximum during the rainy season due to the natural inflow from adjacent areas. In this estuary, the nutrients are mainly controlled by the river discharge, rainfall, wind and tide and exceptionally by la Niña.
\end{abstract}

Key words: Amazon Estuary;Paracauari river; Mesotidal; Marajó Island

\section{Résumé}

Cet article montre la distribution des sels nutritifs des eaux de l'estuaire de Paracauari durant um cycle hydrologique amazônien (2008) et à la fim de la période de la Niña (abril 2008). Cet estuaire reçoit l'influence du climat tropical, et de la méso-marée ( 3 à $4 \mathrm{~m}$ ), semi-diurne. L'échantillonage des sels nutritifs fut réalisé le long de 10 stations, durant 3 périodes: humide (mars), intermédiaire (juin) et moins humide (septembre). Les propriétés physiques des eaux ont été mesurées, em utilizant une sonde multiparamètres, et les sels nutritifs dissous (nitrate, nitrite n-ammoniaque, phosphate et silicate) ont été analizés par spectrophotométrie tandis que les particules en suspension par méthode gravimétrique. Ces concentrations ont subit d'amples modifications tout au long des saisons, en particulier durant la période humide, oú a été registrée La Niña. La temperatura de l'eau (moyenne de $28,58^{\circ} \mathrm{C}$ ) est très homogène, très typique des eaux tropicales. Le $\mathrm{pH}$ a varié de l'état acide $(5,80)$ a alcalin $(7,86)$ et la salinité entre 0,06 a 7,56 avec des valeurs maximales dans l'embouchure, em fonction de l'influence marine. Les eaux sont mal a bien oxigénées, respectivement, durante les saisons humide (2,35 mg.L-1) et moins humide (6,55 mg.L-1). Les concentrations des particules en suspension e des sels nutritifs ont été maximales durant la période humide, em fonction de l'apport naturel provenant des zones adjacentes. Dans l'estuaire de Paracauari, les sels nutritifs são controlés principalmente par l'écoulement fluvial, les précipitations, le vent et la méso-marée.

Mots-clés: Estuaire amazonien, Rivière Paracauari, Zone de Mélange, méso-marée Ile de Marajó.

Palabras clave: Unidades de conservación ambiental, Funciones y servicios ambientales, Geomorfología costera, Licencias ambientales.

(*) Profa. Msc. da Faculdade de Oceanografia da Universidade Federal do Pará - Cidade Universitária Prof. José da Silveira Netto, CEP 66075-110. Belém (PA), Brasil. Tel.: (+ 55 91) 3089-0816 - sury@ufpa.br

(**) Prof. Dr. da Universidade Federal do Pará - Cidade Universitária Prof. José da Silveira Netto, Caixa Postal 8617. Av. Augusto Corrêa, 01, CEP 66075-110. Belém (PA), Brasil. Tel.: (+55 91) 3201-7747 - robrini@ufpa.br

(***) Doutorando no Programa de Pós-graduação em Geologia e Geoquímica da Universidade Federal do Pará - Cidade Universitária Prof. José da Silveira Netto, CEP 66075-110. Belém (PA), Brasil. Tel.: (+55 91) 3201-7747 - igorcharles@ufpa.br 


\section{INTRODUÇÃO}

Os estuários da costa norte do Brasil são ecossistemas dinâmicos, constituem locais de refúgio, reprodução e crescimento de inúmeras espécies animais (AVELINE, 1980), apresentam elevada produtividade primária (BRAGA et al., 2000; PEREIRA FILHO et al., 2001), importância sócio-ambiental e estão inseridos em uma complexa rede hidrográfica, tendo como estuário principal o Amazonas (BARTHEM, 1985).

O comportamento dos nutrientes nos estuários envolve uma intima relação entre sedimentos particulados e dissolvidos de fundo e os processos de adsorção, desorção, transporte e transformações biológicas (CORRELL, 1981; JORDAN et al., 1991). Para entender essas relações e seus fatores controladores nas águas estuarinas amazônicas, diversas pesquisas estão sendo desenvolvidas. Pode-se citar: Dittmar e Lara (2001) analisaram a dinâmica dos nutrientes inorgânicos e da matéria orgânica no manguezal do estuário Caeté-PA; Cohen et al. (2004) relacionaram o grau de inundação e elevação das marés e a frequência do manguezal aos dados existentes das análises físico-químicas e de fosfato nas águas de um canal no norte do Brasil; Santos et al. (2008) estudaram a influência dos rios Amazonas e Pará sobre a distribuição dos parâmetros físico-químicos, e da biomassa do fitoplâncton na plataforma continental e áreas oceânicas adjacentes; Alves (2010) estudou a produtividade primária e hidrologia do sistema flúvio-estuarino do Arari na Ilha do Marajó-PA; e Melo (1998) estudou o comportamento biogeoquímico de nutrientes no estuário do rio Bacanga-MA.

O estuário do rio Paracauari, na margem leste da Ilha do Marajó, Estado do Pará, está situado em uma interface dinâmica na foz do rio Pará, apresentando características particulares em relação a outros sistemas estuarinos. Este estuário é fortemente influenciado pelas variações sazonais, descarga hídrica e sólida das drenagens regionais, ventos, marés e correntes associadas (Figura 1). Este artigo tem como objetivo compreender o comportamento espacial dos parâmetros abióticos (temperatura, salinidade, $\mathrm{pH}$, oxigênio dissolvido e material particulado em suspensão), dos nutrientes dissolvidos (nitrato, nitrito, N-amoniacal, fosfato e silicato) no estuário do rio Paracauari, durante o ciclo hidrológico de 2008 (período chuvoso - março, intermediário - junho e menos chuvoso setembro), fortemente influenciado pela La Niña durante os primeiros 4 meses.
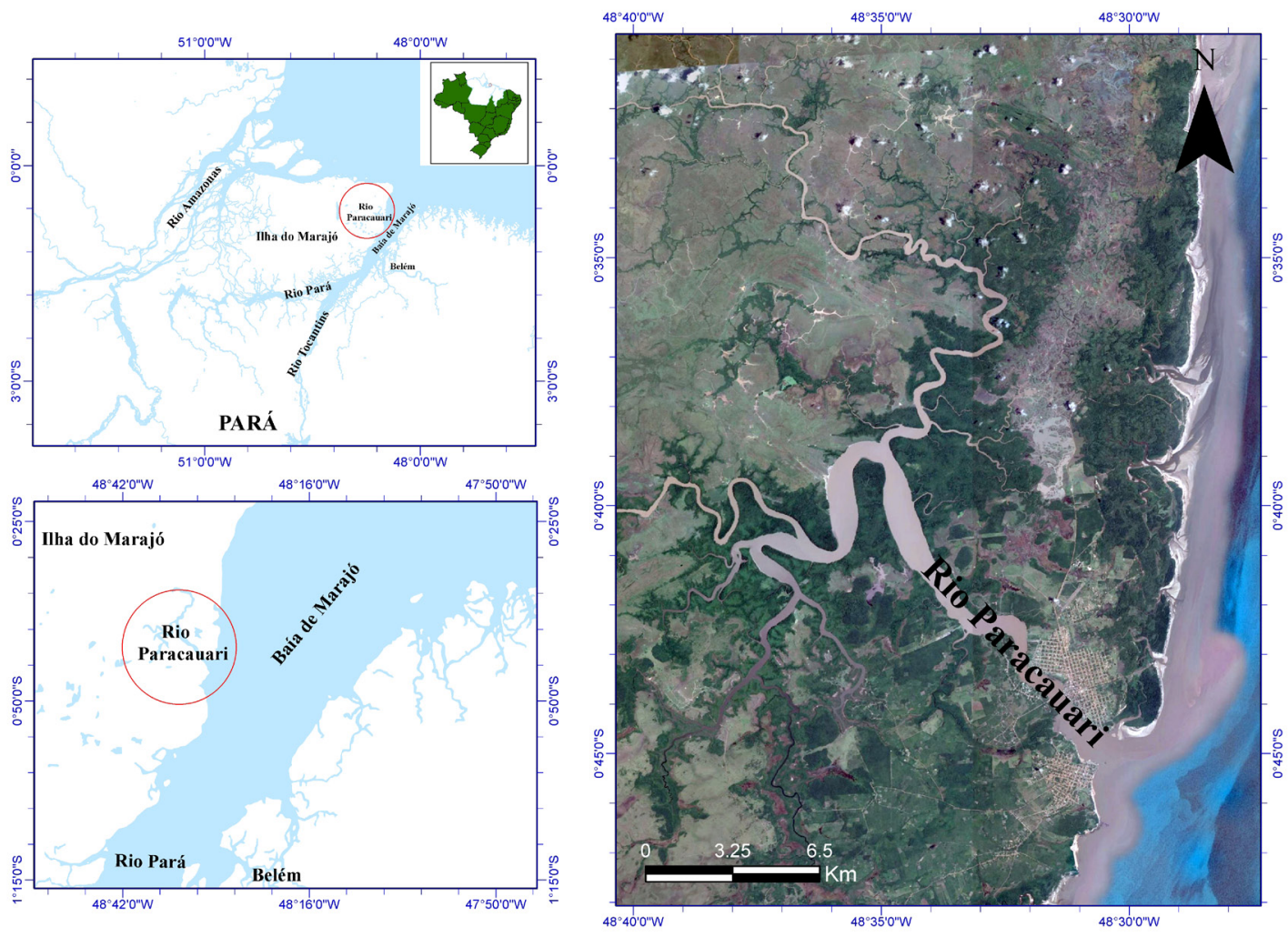

Figura 1 - Mapa de localização do estuário do rio Paracauari, Ilha do Marajó - PA. 


\section{MATERIAL E MÉTODOS}

\section{Área de Estudo}

O estuário do rio Paracauari banha a divisão dos municípios de Soure e Salvaterra e representa uma região estratégica que sustenta diversas atividades socioeconômicas na margem leste da Ilha do Marajó-PA.

Nesse estuário ocorrem principalmente os sedimentos do Grupo Barreiras e são identificadas duas unidades morfoestruturais: o planalto costeiro predominante no município de Salvaterra e a planície costeira em Soure (FRANÇA e SOUZA FILHO, 2006). Quanto à vegetação, observam-se manguezais associados a espécies de várzea (ALMEIDA, 1995), sendo que os manguezais são predominantes próximos à foz, enquanto que a várzea e vegetação de terra firme à montante do estuário.

A extensão desse estuário é de aproximadamente $50 \mathrm{~km}$ e sua bacia de drenagem totaliza uma área de $608 \mathrm{~km} 2$ pertencente a Bacia Hidrográfica Amazônica juntamente com as demais bacias hidrográficas dos rios existentes na Ilha do Marajó (ANA, 2007).

Apresenta-se altamente meandrante com desvios moderados de seu curso d'água, sendo que, para sua montante, flui na direção E-W, enquanto que para sua jusante, sofre alta inflexão (i.e., ângulo de $90^{\circ}$ ), fluindo para N-S e SE (SOUZA e ROSSETTI, 2009).

Por ser um estuário situado em uma região tropical, é caracterizado por baixas variações de insolação e temperatura, um clima tropical úmido, com temperatura média anual de $27^{\circ} \mathrm{C}$, pluviosidade média de $2.500 \mathrm{~mm} /$ ano e dois períodos sazonais distintos: chuvoso que ocorrem de dezembro a maio e menos chuvoso de julho a novembro. A velocidade média anual do vento é de $2,4 \mathrm{~m} / \mathrm{s}$ e a direção predominante é de nordeste (INMET, 2008). Durante o período chuvoso, o volume pluviométrico mensal foi acima da normal climatológica (1961-2009) (BALLMAN et al., 2012). Isto significa que La Niña 2007/2008 contribuiu para o aumento da precipitação na Amazônia Oriental, que atingiu aproximadamente $650 \mathrm{~mm}$ no mês de março.

As águas deste estuário sofrem incursão semi-diurna de meso a macromaré (3 a 6 m) (DHN, 2008).

\section{Amostragem}

Foram realizadas amostragens de água ao longo do estuário do rio Paracauari em superfície e fundo $(10 \mathrm{~m})$, em dez pontos de coleta equidistantes entre si em $1,5 \mathrm{~km}$, sendo que o ponto 1 (P1) está localizado na foz, com maiores influencias das águas salobras da baia de Marajó e o ponto 10 (P10) a montante do estuário, com maior influência fluvial (Figura 2). Os pontos de amostragem foram posicionados com um receptor GPS (Global Position System).

As três campanhas oceanográficas foram efetuadas durante o ciclo hidrológico de 2008, levando-se em consideração a sazonalidade climática da região e a influência da maré de sizígia. A primeira campanha foi realizada em março, período chuvoso, coincidindo com o máximo de precipitação pluviométrica $(650 \mathrm{~mm})$ no final do período de La Niña e o mínimo de insolação; a segunda ocorreu em junho coincidindo com o período de transição entre o período chuvoso e menos chuvoso $(260 \mathrm{~mm})$; e a terceira em setembro, correspondente ao período menos chuvoso, com um mínimo de precipitação $(10 \mathrm{~mm})$.

A amostragem ao longo do estuário do rio Paracauari ocorreu sistematicamente no sentido jusante-montante na maré vazante. Em cada ponto coletou-se amostras de água superficial e de fundo com o auxílio de uma garrafa oceanográfica do tipo Van Dorn. Em seguida, analisou-se in situ a temperatura, $\mathrm{pH}$, condutividade elétrica e salinidade através de um multianalisador da marca Hanna, modelo 9828; oxigênio dissolvido (OD) foi determinado pelo método iodométrico clássico de Winkler, descrito em Strickland e Parsons (1972); e material particulado em suspensão (MPS) através do método gravimétrico descrito em Baumgarten et al. (1996). 


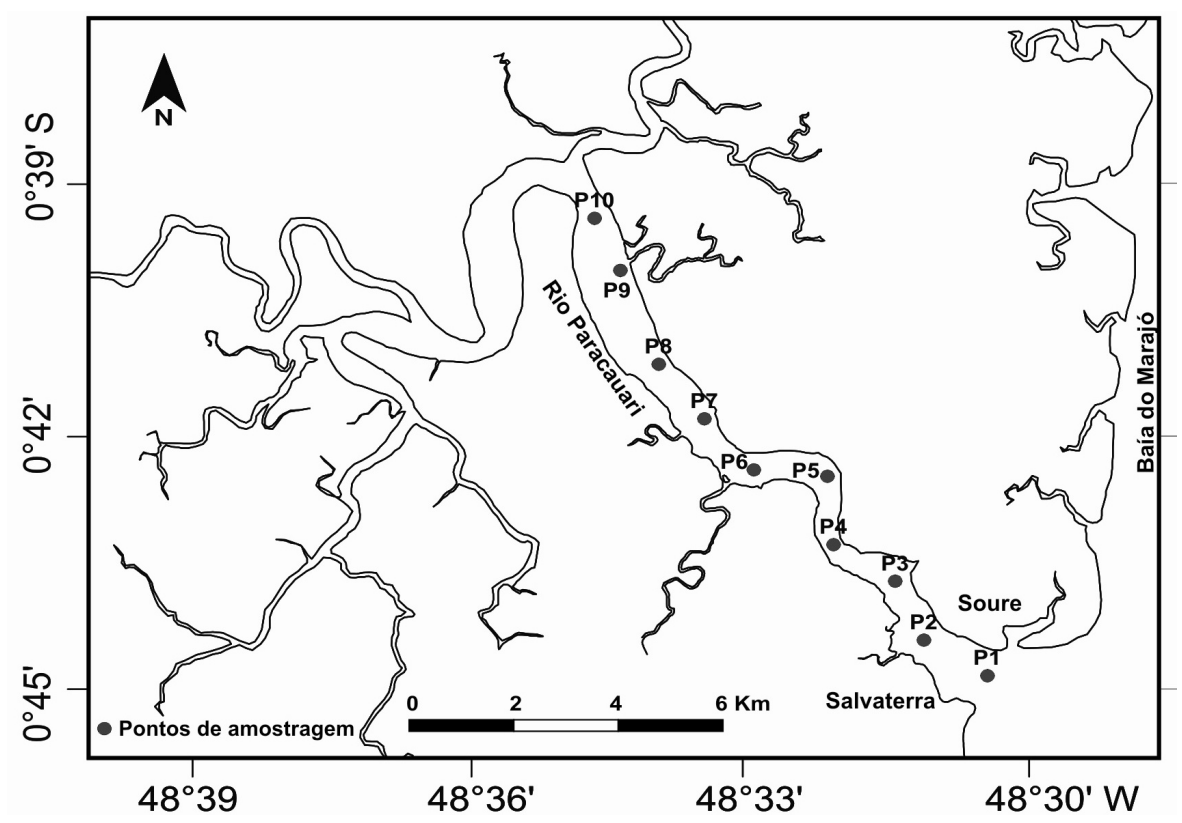

Figura 2 - Localização dos pontos de amostragem no estuário do rio Paracauari, Ilha do Marajó - PA.

Para determinação do nitrato, nitrito, $\mathrm{N}$-amonical, fosfato e silicato foram filtrados $500 \mathrm{ml}$ das amostras de água utilizando filtros de $0.45 \mu \mathrm{m} \mathrm{GF} / \mathrm{F} 47 \mathrm{~mm}$. As amostras de água foram armazenadas em frascos de polietileno e refrigeradas para posterior análise no Laboratório do GEMC e Hidroquímica da Universidade Federal do Pará. As análises dos nutrientes foram realizadas em duplicata de acordo com a metodologia descrita em Grasshoff et al. (1983).

\section{RESULTADOS}

Alguns parâmetros estatísticos descritivos das variáveis em estudo no estuário do rio Paracauari, durante o ciclo hidrológico de 2008 estão descritos na Tabela 1.

No estuário do rio Paracauari, os parâmetros abióticos estudados apresentaram variações sazonais (período chuvoso, intermediário e menos chuvoso) e longitudinais (montante e jusante). $\mathrm{Na}$ coluna d'água (superfície e $10 \mathrm{~m}$ ), as médias foram semelhantes em ambas as profundidades, assim, não verificou-se estratificação vertical no estuário durante os períodos estudados.

Dentre os períodos estudados, os valores máximos de temperatura da água, salinidade, $\mathrm{pH}$ e oxigênio dissolvido ocorreram durante o período menos chuvoso.

Sazonalmente a temperatura da água variou entre $28,18^{\circ} \mathrm{C}$ e $29,18{ }^{\circ} \mathrm{C}$ (média de $28,58{ }^{\circ} \mathrm{C}$ e variação máxima de $1,20^{\circ} \mathrm{C}$ ), confirmando a homogeneidade térmica das águas tropicais. $\mathrm{O} \mathrm{pH}$ variou entre ácido e alcalino (5,80 e 7,86) (Figura 3a), a salinidade entre 0,06 e 7,56 (Figura 3b), e o oxigênio dissolvido entre 2,35 e 6,55 mg.L-1 (Figura 3c).

Longitudinalmente, a temperatura da água não variou significativamente, com maiores amplitudes $\left(0,86^{\circ} \mathrm{C}\right)$ no período intermediário. A salinidade, o $\mathrm{pH}$ e o $\mathrm{OD}$, mostraram um gradiente decrescente da foz à montante, com excessão da salinidade no período chuvoso que apresentou um gradiente crescente $(0,06$ a 0,12$)$ neste mesmo sentido. Nos períodos intermediário e menos chuvoso a variação decrescente desse parâmetro foi de 1,63 a 0,63 e 7,56 a 5,84, respectivamente.

$\mathrm{O} \mathrm{pH}$ decresceu longitudinalmente de básico à ácido nos períodos chuvoso $(6,5$ a 5,8$)$ e intermediário $(7,26$ a 6,64$)$ no sentido foz-montante, e no período menos chuvoso decresceu, porém em menor escala, no mesmo sentido, de 7,73 a 7,32 mg.L-1.

A variação longitudinal nas concentrações de OD foram bastante significativas nos períodos chuvoso (4,40 a 2,85 mg.L-1) e intermediário (5,45 a 4,22 mg.L-1), no menos chuvoso, contudo, essa variação foi menor (6,55 a 6,18 mg.L-1).

Tabela 1 - Estatística descritiva (mínimo, máximo, mediana) dos dados abióticos (temperatura, 
salinidade, OD, $\mathrm{pH}, \mathrm{MPS}$, nitrato, nitrito, N-amoniacal, fosfato e silicato) na coluna d'água do estuário do rio Paracauari - Ilha do Marajó

\begin{tabular}{|c|c|c|c|c|c|c|c|c|c|}
\hline \multirow[b]{2}{*}{ Parâmetros } & \multicolumn{3}{|c|}{ Chuvoso } & \multicolumn{3}{|c|}{ Intermediário } & \multicolumn{3}{|c|}{ Menos chuvoso } \\
\hline & Mín. & Máx. & Med. & Mín. & Máx. & Med. & Mín. & Máx. & Med. \\
\hline & \multicolumn{9}{|c|}{ Superfície } \\
\hline Temperatura & 28,18 & 28,41 & 28,31 & 28,52 & 29,35 & 28,85 & 28,32 & 29,18 & 28,68 \\
\hline $\mathrm{pH}$ & 5,80 & 6,50 & 6,18 & 6,64 & 7,26 & 6,99 & 7,30 & 7,86 & 7,53 \\
\hline MPS & 77,33 & 115,67 & 99,43 & 19,33 & 63,00 & 34,03 & 12,14 & 18,91 & 15,03 \\
\hline Salinidade & 0,06 & 0,15 & 0,10 & 0,63 & 1,63 & 1,24 & 5,84 & 7,56 & 7,13 \\
\hline OD & 2,85 & 4,40 & 3,53 & 4,22 & 5,45 & 4,99 & 6,18 & 6,55 & 6,38 \\
\hline Nitrato & 5,16 & 8,23 & 6,45 & 0,81 & 5,65 & 2,28 & 0,65 & 4,94 & 2,24 \\
\hline Nitrito & 0,01 & 0,10 & 0,05 & 0,01 & 0,11 & 0,03 & 0,01 & 0,07 & 0,03 \\
\hline N-amoniacal & 0,40 & 0,56 & 0,48 & 0,26 & 0,78 & 0,43 & 0,15 & 0,77 & 0,37 \\
\hline Fosfato & 0,77 & 1,08 & 0,92 & 0,15 & 0,54 & 0,33 & 0,11 & 0,94 & 0,30 \\
\hline \multirow[t]{2}{*}{ Silicato } & 41,46 & 83,10 & 60,76 & 23,95 & 49,85 & 35,53 & 14,98 & 31,13 & 20,20 \\
\hline & \multicolumn{9}{|c|}{ FUNDO (10m) } \\
\hline Temperatura & 28,27 & 28,43 & 28,37 & 28,46 & 28,93 & 28,72 & 28,35 & 28,72 & 28,6 \\
\hline $\mathrm{pH}$ & 5,53 & 6,4 & 6,09 & 6,78 & 7,22 & 7,06 & 7,32 & 7,64 & 7,48 \\
\hline MPS & 62,00 & 98,67 & 86,26 & 30,67 & 56,33 & 42,07 & 12,51 & 17,90 & 15,40 \\
\hline Salinidade & 0,06 & 0,15 & 0,10 & 0,78 & 1,94 & 1,37 & 6,49 & 7,59 & 7,24 \\
\hline OD & 2,60 & 4,15 & 3,24 & 4,48 & 5,48 & 4,96 & 6,18 & 6,52 & 6,36 \\
\hline Nitrato & 5,10 & 8,90 & 6,60 & 1,10 & 3,55 & 2,14 & 0,86 & 4,21 & 1,95 \\
\hline Nitrito & 0,01 & 0,11 & 0,06 & 0,01 & 0,09 & 0,02 & 0,01 & 0,69 & 0,11 \\
\hline N-amoniacal & 0,36 & 0,56 & 0,45 & 0,11 & 0,67 & 0,40 & 0,10 & 0,27 & 0,20 \\
\hline Fosfato & 0,74 & 0,97 & 0,86 & 0,18 & 0,45 & 0,29 & 0,10 & 0,84 & 0,22 \\
\hline Silicato & 43,53 & 81,30 & 59,54 & 21,00 & 43,00 & 31,23 & 13,00 & 29,00 & 18,87 \\
\hline
\end{tabular}

O material particulado em suspensão, sazonalmente, variou entre 12,14 e 115,67 mg.L-1 com concentrações máximas no período chuvoso (Figura 3d). Longitudinalmente, teve um gradiente crescente da foz à montante, com variações mais significativas no período chuvoso $(77,33$ a 115,67 mg.L-1) e intermediário (19,33 a 63,00 mg.L-1), e menos significativa no menos chuvoso (12,51 e 17,90 mg.L-1).

Os nutrientes dissolvidos (nitrato, nitrito, $\mathrm{N}$-amonical, fosfato e silicato) variaram sazonalmente e longitudinalmente. Na coluna d'água, contudo, suas concentrações foram homogêneas, ou seja, sem amplas variações entre superfície e fundo.

Sazonalmente, as concentrações máximas dos nutrientes estudados ocorreram no período chuvoso e mostraram um decréscimo nos outros períodos (intermediário e menos chuvoso).

O nitrato variou entre 0,64 e 8,22 $\mu$ M.L-1, com média de $6,45 \mu \mathrm{M}$.L-1 no período chuvoso e um decréscimo significante para 2,28 $\mu$ M.L-1 e 2,24 $\mu$ M.L-1 no período intermediário e menos chuvoso, respectivamente (Figura 4a). As concentrações de nitrito e n-amoniacal foram as menores dentre os compostos nitrogenados analisados e não variaram expressivamente entre os períodos estudados, sendo que o nitrito variou entre 0,01 e $0,09 \mu \mathrm{M}$.L-1, com médias decrescendo de 0,05 a $0,03 \mu$ M.L-1, e o n-amoniacal variou entre 0,15 e $0,78 \mu$ M.L-1 com médias decrescendo de 0,48 a $0,37 \mu$ M.L-1 (Figura $4 \mathrm{~b}$ e 4c). A média de fosfato decresceu de 0,92 a 0,30 $\mu$ M.L-1 entre o período chuvoso e menos chuvoso e sua variação sazonal foi de 0,11 e 1,08 $\mu$ M.L-1 (Figura 4d).O silicato foi o nutriente que variou de forma mais expressiva (14,98 a 83,10 $\mu \mathrm{M}$.L-1), com média de 60,76 $\mu$ M.L-1 no período chuvoso, a qual decresceu para 35,53 $\mu$ M.L-1 no intermediário e para 20,20 $\mu$ M.L-1 no menos chuvoso (Figura 4e). 

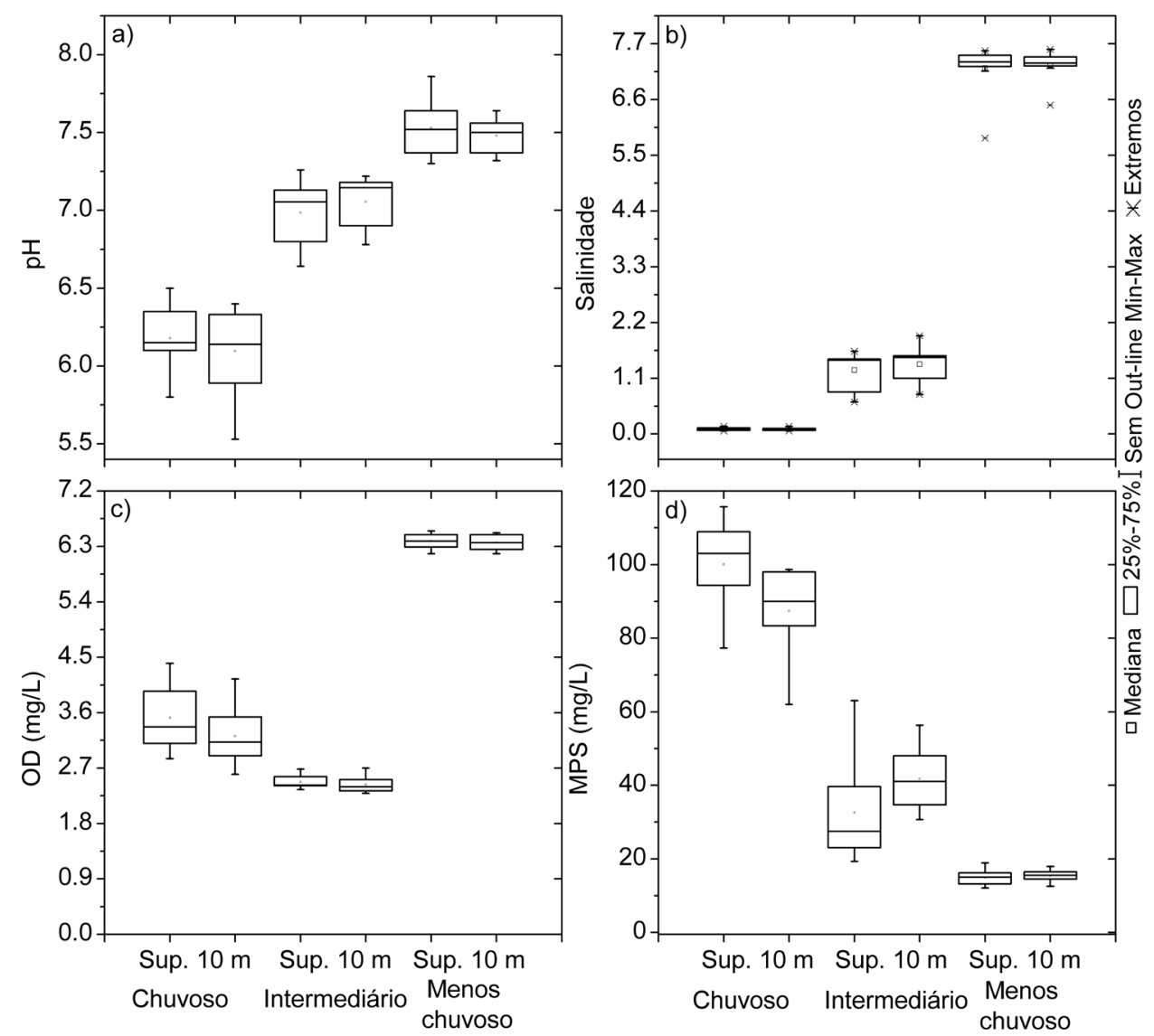

Figura 3 - Variação sazonal dos parâmetros abióticos analisados no estuário do rio Paracauari (Ilha do Marajó-PA): a) $\mathrm{pH}$; b) salinidade; c) OD e d) material particulado em suspensão

Longitudinalmente, a variação dos nutrientes não foi regular, porém, as menores concentrações em geral ocorreram na foz, principalmente no período menos chuvoso, quando há maior diluição das águas continentais pelas águas salobras, pobres em nutrientes, provenientes da baía do Marajó. Somente o fosfato mostrou um padrão diferente, ou seja, próximo aos municípios de Soure e Salvaterra, na foz, ocorreram as concentrações mais elevadas (1,08 $\mu$ M.L-1 no chuvoso, $0,54 \mu$ M.L-1 no intermediário e $0,94 \mu \mathrm{M}$.L-1 no menos chuvoso), e as menores à montante $(0,77 \mu \mathrm{M}$.L-1 no chuvoso, $0,15 \mu$ M.L-1 no intermediário e $0,11 \mu$ M.L-1 no menos chuvoso).

Os compostos nitrogenados demonstraram um gradiente crescente da foz a montante. O nitrato variou de 5,16 a 8,22 $\mu$ M.L-1 no período chuvoso, enquanto que nos outros períodos, com o decréscimo nas concentrações de nitrato na coluna d'água, a variação foi de 0,80 a $5,64 \mu \mathrm{M}$.L-1 e 0,64 a 4,93 $\mu \mathrm{M} . \mathrm{L}-1$, nos período intermediário e menos chuvoso, respectivamente. As concentrações mínimas de nitrito foram iguais $(0,01 \mu \mathrm{M} . \mathrm{L}-1)$ em todos os períodos e máximas variaram de 0,09 $\mu$ M.L-1 no período chuvoso, $0,11 \mu \mathrm{M}$.L-1 no intermediário e $0,07 \mu \mathrm{M}$.L-1 no menos chuvoso. As menores variações de $\mathrm{n}$-amoniacal ocorreram no período chuvoso ( 0,44 a $0,55 \mu \mathrm{M} . \mathrm{L}-1)$ e as maiores no menos chuvoso $(0,15$ a $0,77 \mu$ M.L-1).

As concentrações de silicato foram as que mais variaram nos diversos períodos: 41,46 a 83,10 $\mu$ M.L-1 no chuvoso, 23,95 a 49,85 $\mu$ M.L-1 no intermediário e 14,98 a 31,13 $\mu$ M.L-1 no menos chuvoso. 

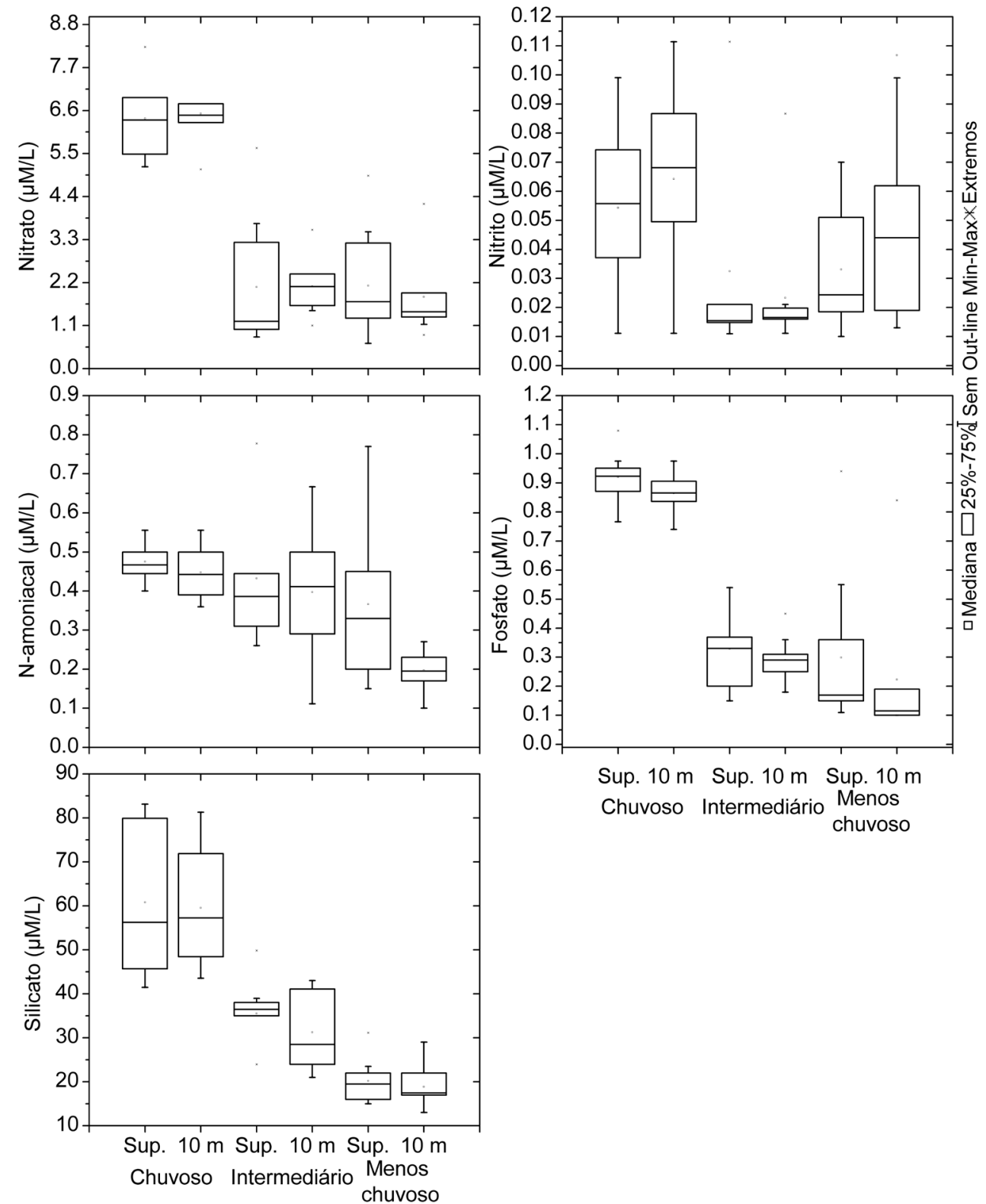

Sup. $10 \mathrm{~m}$ Sup $10 \mathrm{~m}$ Sup $10 \mathrm{~m}$

chuvoso

Figura 4 - Variação sazonal dos nutrientes inorgânicos dissolvidos no estuário do rio Paracauari (Ilha do Marajó PA): a) nitrato; b) nitrito; c) n-amoniacal; d) fosfato; e) silicato.

\section{DISCUSSÃO}

Os estuários apresentam variações espaciais e temporais continuas (ELLIOTT e MCLUSKY, 2002). No estuário do rio Paracauari há uma variação dos parâmetros estudados em escala temporal de acordo com as estações do ano em associação com o clima da região amazônica, e variações longitudinais influenciadas principalmente pela descarga fluvial, pelos efeitos da precipitação, do vento, da maré e das correntes de maré (MCLUSKY, 1989; SHAEFFER-NOVELLI, 1982; UNDERWOOD e KROMKAMP, 1999). Essas variáveis fazem com que o estuário apresente-se "segmentado" durante os períodos analisados (chuvoso, intermediário e menos chuvoso) com relação aos principais parâmetros analisados.

As águas do estuário do rio Paracauari apresentaram elevada estabilidade térmica temporal, típica da águas estuarinas tropicais, a qual depende do período e grau de insolação e variações meteorológicas, sendo estas condicionadas por períodos de maior ou menor nebulosidade (Flores Montes 1996). Entretanto na coluna d'água a homogeneização da temperatura foi possivelmente causada pelo grande volume de água descarregada, à turbulência consequência do vento e pelas correntes de maré (SANTOS et al., 1986). 
Essa estabilidade térmica é típica das águas estuarinas tropicais, que podem variar de $24^{\circ}$ a $30{ }^{\circ} \mathrm{C}$, e está relacionada ao gradiente de salinidade que pode aumentar a temperatura em até $4{ }^{\circ} \mathrm{C}$ principalmente no período menos chuvoso (BRADLEY e PHILIP, 1999).

As medidas de salinidade refletem, sazonalmente e longitudinalmente, a influência das águas continentais, menos salinas, durante o período chuvoso e intermediário, e das águas costeiras da Baía do Marajó, mais salinas, no menos chuvoso.

No período chuvoso, o comportamento diferenciado, com maiores concentrações de sais a montante do estuário, ocorreu provavelmente devido à exportação de sais retidos nas várzeas, após alagamento, para o estuário. Assim, entende-se que as várzeas que margeiam o estuário do rio Paracauari desempenham um importante papel regulador nas concentrações de sais dessas águas. Nos demais períodos, quando a vazão dos rios diminui significantemente, as águas costeiras (mais salinas) penetram o estuário cerca de $40 \mathrm{~km}$ em relação à foz. Em outros estuários paraenses, com a penetração das águas costeiras, a salinidade atinge índices mais elevados (21 a 38) no final do período menos chuvoso (LARA e DITTMAR, 1999).

Através dos índices de salinidade, é possível sugerir que o estuário do rio Paracauari é verticalmente homogêneo, podendo ser classificado como estuário de águas oligohalinas no período chuvoso e intermediário e mesohalinas no período menos chuvoso.

As concentrações de OD nesse estuário foram influenciadas pelas altas taxas de sedimentação que aumentam a transparência da água, favorecem a penetração solar, principalmente no período menos chuvoso, e consequentemente intensifica a atividade fotossintética, a qual influencia diretamente na elevação das concentrações desse gás. Na foz do estuário do rio Amazonas, observou-se concentrações de OD semelhantes ao estuário do rio Paracauari: como 5,23 mg.L-1 detectado por Magliocca (1971) e 6,54 mg.L-1 por Santos et al. (2008).

As mudanças sazonais de $\mathrm{pH}$ são consequência da redução do efeito acidificante do gás carbônico livre e dissolvido e, em grande parte, da presença de ácidos orgânicos que são transportados para o estuário principalmente no período chuvoso. As variações sazonais e longitudinais de $\mathrm{pH}$ coincidiram com as de OD. Essa correlação também foi observada por Demaster et al. (1996) em seu estudo nas águas superficiais da plataforma continental do Amazonas.

A grande concentração de material em suspensão no estuário do rio Paracauari provoca um aspecto barrento nas águas, e é consequência do escoamento superficial de material particulado e dissolvido para o rio durante o período chuvoso. Com a diminuição dos índices pluviométricos, no período menos chuvoso, há também a diminuição da vazão do rio, e a carga de material em suspensão passa a ser controlada principalmente pela energia dos ventos e marés, por isso observa-se o oposto, isto é, águas mais transparente devido à maior penetração de águas costeiras, mais límpidas.

As reduções sazonais entre o período chuvoso e menos chuvoso e as variações longitudinais nas concentrações de nutrientes já era um comportamento esperado, pois esse estuário está sujeito a intensa influencia: da floresta equatorial, rica em biodiversidade, notadamente em bactérias oxidantes (produzem nitrato) e redutoras (produzem predominantemente amônia); das águas costeiras, menos enriquecidas de nutrientes; e do consumo pelo fitoplâncton.

O nitrato foi a espécie predominante entre os compostos nitrogenados, que apresentou concentrações bastante estáveis e distribuiu-se linearmente em todo o estuário. Sua variação longitudinal pode está relacionada ao processo de nitrificação, com o nitrato resultante da conversão do n-amoniacal ou nitrito, tornando as concentrações desta forma relativamente mais baixas. Este processo de oxidação pode ter sido facilitado pelas concentrações de OD razoavelmente disponível. Além disso, as concentrações de nitrato podem ser controladas pelo aporte dos sedimentos do fundo, uma vez que a montante do estuário tem-se as menores profundidades.

As concentrações de fosfato, provavelmente influenciadas pela contribuição dos particupados oriundos do escoamento fluvial, ocorreram abaixo das encontradas em outros estuários tropicais. Por exemplo, Lara e Dittmar (1999) verificaram concentrações de fosfato variando entre 1,5 a 5,0 $\mu$ M.L-1 em um canal de maré (Bragança-PA) durante o período seco, e atribuiram esses baixas 
concentrações a absorção por produtores primários e bactérias, que utilizam esse nutriente durante o processo fotossintético.

Outros processos associados que poderiam estar afetando na distribuição de compostos nitrogenados e fosfatados seriam: mistura vertical produzida pelo vento; sedimentação de compostos fosfatados em associação com outros compostos; e remineralização da matéria orgânica.

O silicato, devido a sua origem terrígena, é o nutriente inorgânico com maiores concentrações nos estuários. É utilizado pelas microalgas do grupo das diatomáceas e dos silicoflagelados para formação das suas frústulas (FERNANDES, 1994).

Ainda, as elevadas concentrações desse nutriente podem estar ligadas a processos físicos de diluição das águas fluviais. Assim, o motivo das elevadas concentrações de silicato nas águas do estuário do rio Paracauari, principalmente durante o período chuvoso, é a intensa lixiviação dos sedimentos através do intemperismo nas margens do estuário e do manguezal.

O comportamento sazonal e espacial de silicato observado no estuário do rio Paracauari é semelhante ao encontrado por outros autores, por exemplo: em relação à variação sazonal, Bradley e Philip (1999) afirmam que as maiores concentrações de silicato nos estuários tropicais foram encontradas durante o período chuvoso. Já em relação à distribuição longitudinal do silicato, Melo (1998) ao analisar as águas do estuário do rio Bacanga - MA, encontrou os maiores valores (200 $\mu \mathrm{M}$.L-1) a montante do rio e os menores (20 a $50 \mu \mathrm{M}$.L-1) a jusante em função do aumento da salinidade. Vale ressaltar que as concentrações de silicato nas águas amazônicas variam entre 2 a $187 \mu$ M.L-1 (EDMOND et al., 1983).

Através dos resultados analíticos, pode-se propor que o suprimento de nitrogênio, fósforo e silicato é feito principalmente pela descarga fluvial e pela liberação do sedimento. Esses parâmetros admitem um padrão não conservativo, ou seja, depende diretamente de processos biológicos realizados pela biomassa fitoplanctônica na zona eufótica, ou por processos físicos, como advecção.

Em relação à escala de concentração desses nutrientes, no estuário do rio Paracauari, o silicato foi o mais concentrado seguido pelo nitrato, depois pelo fosfato e por fim pelo $\mathrm{N}$-amoniacal e nitrito. Essa escala de concentração também foi observada por Noriega et al. (2005) no sistema estuarino de Barra das Jangadas (Pernambuco - Brasil). Apesar da descarga do rio ter a tendência de aumentar fortemente as concentrações de $\mathrm{N}$-amoniacal, na área estudada este fato não foi tão evidenciado, o que pode ser indicativo do aumento do processo de oxidação (NORIEGA et al., 2005). A redução nas concentrações de $\mathrm{N}$-amoniacal também representa a consequência da assimilação pelo fitoplâncton.

\section{Anãlise Multivariada}

A análise de componentes principais com todos os parâmetros abióticos obtidos na superfície e no fundo $(10 \mathrm{~m})$ mostra que as duas primeiras componentes (Fator 1 e Fator 2) juntas descrevem $76,71 \%$ da variância original (Figura 5a). O Fator 1 explicou 65,06 \% da variância total e pode ser interpretado como um contraste entre, de um lado, temperatura $(0,55), \mathrm{pH}(0,96)$, salinidade $(0,82)$ e OD $(0,94)$ e, do outro lado, MPS $(-0,96)$, nitrato $(-0,88)$, nitrito $(-0,44)$, $\mathrm{N}$-amoniacal $(-0,53)$, fosfato $(-0,85)$ e silicato $(-0,87)$.

Essa inversão indica que os dois grupos de parâmetros se correlacionaram negativamente. Para a maioria dos parâmetros, essa conclusão é bastante razoável, pois explica, por exemplo, na foz, a variação inversa entre os maiores índices de salinidade e os menores de silicato.

Essa correlação sugere que as concentrações desse nutriente no estuário do rio Paracauari são predominantemente controladas pelas emissões das fontes de origem continental, que são integradas e transferidas para os estuários através do fluxo fluvial (WHITE et al., 2004), e podem ser oriundas de fontes naturais, provavelmente localizadas em áreas a montante da bacia de drenagem.

Do mesmo modo, essa correlação linear negativa entre silicato e salinidade sugere que o comportamento não conservativo dos nutrientes neste estuário seja resultado de processos como a retenção deste elemento nos sedimentos estuarinos, a transformação em outras espécies químicas ou a fixação biológica (PITKÄNEN et al., 1993; LIU et al., 2005). 
Outra conclusão esperada foi as correlações de $\mathrm{pH}$, salinidade e material particulado em suspensão apresentam um comportamento conservativo, ou seja, estão sujeitos tão somente aos processos de diluição e mistura da água salgada com a água doce. Enquanto que parâmetros como $\mathrm{OD}, \mathrm{N}$-amoniacal, nitrito, nitrato, fosfato e silicato se comportam de maneira não-conservativa, logo estão sujeitos ao consumo biológico pelo fitoplâncton e bactérias.

Os escores demonstram uma variação sazonal dos valores medidos na coluna d'água do estuário do rio Paracauari (Figura 5b). As amostras de água referentes ao período chuvoso estão posicionadas na direção do $4^{\circ}$ quadrante, enquanto que as do intermediário e menos chuvoso encontram-se no $1^{\circ}$ e $2^{\circ}$ quadrantes. Não há variações significativas na coluna d'água, principalmente devido aos processos de mistura ocasionados pela forte influência de processos físicos como as marés a as fortes turbulências.

A análise de componentes principais mostra a influência da água continental sobre a distribuição dos parâmetros abióticos estudados, durante o período chuvoso, nos dois níveis de profundidade, e das águas salobras durante o período intermediário e menos chuvoso.
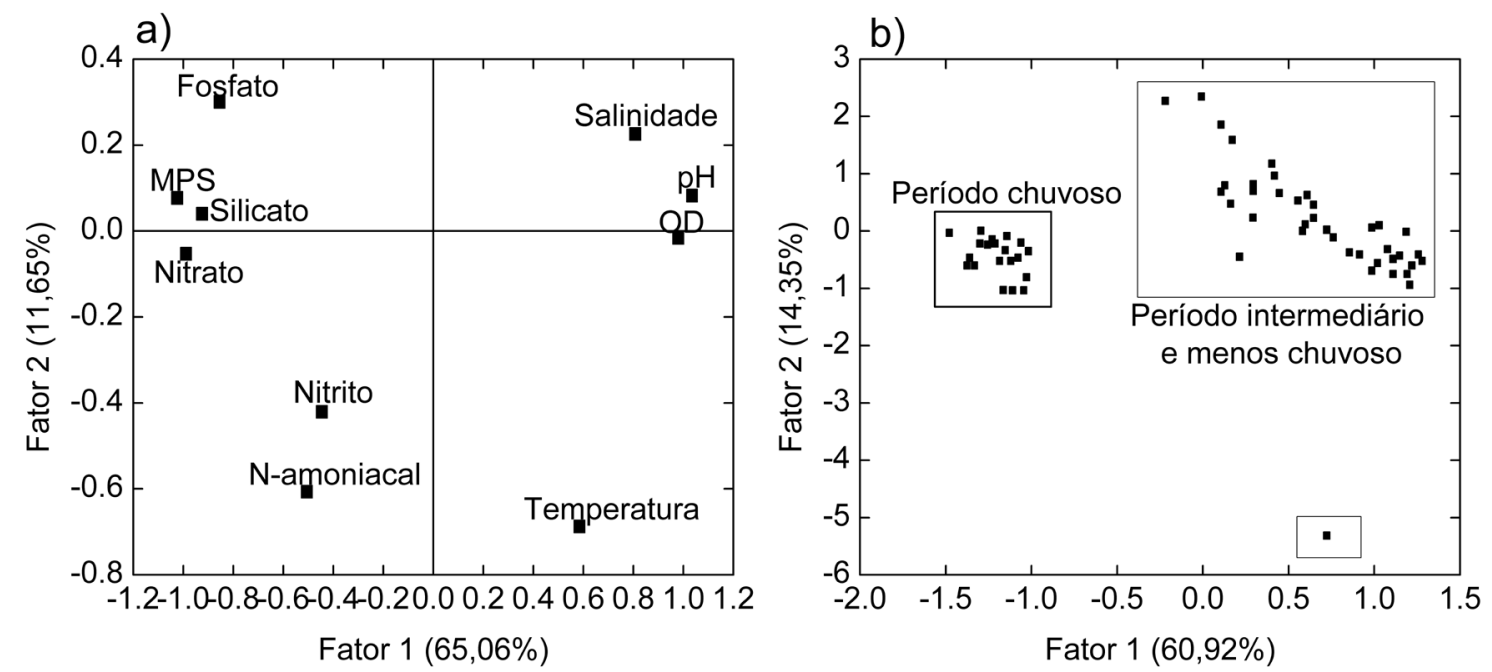

Figura 5 - a) Pesos das variáveis nas duas primeiras componentes principais e b) escores nas duas primeiras componentes principais na análise dos parâmetros abióticos das águas estuarinas do rio Paracauari - Ilha do Marajó, em três períodos sazonais distintos.

\section{CONSIDERAÇÕES FINAIS}

O estuário do rio Paracauari pode ser classificado como um estuário de planície costeira, segundo os critérios geológicos, e sua circulação apresenta um comportamento nitidamente regulado por marés. É um estuário verticalmente homogêneo, porém, apresenta-se "segmentado" (com variações longitudinais) em relação aos principais parâmetros estudados.

Os parâmetros estudados variam em escala temporal de acordo com as estações do ano em associação com o clima da região amazônica. Esta variação é mais nítida durante o período chuvoso, pois ocorre um máximo de entrada alóctone através do escoamento superficial e sub-superficial. Além disso, verificaram-se alterações longitudinais nos parâmetros analisados e na dinâmica das zonas do estuário do rio Paracauari as quais são controladas principalmente pela descarga fluvial, pelos efeitos da precipitação, do vento, da maré, das correntes de maré, pela influência do manguezal e da várzea, das águas costeiras da Baía do Marajó e, no caso dos nutrientes essenciais, podem-se considerar também as trocas com a atmosfera.

A salinidade foi influenciada principalmente pelas águas costeiras da Baía do Marajó, o OD pelas trocas com a atmosfera e produção primária, e o material em suspensão pela ação das correntes de maré. O suprimento de nutrientes provém da descarga fluvial e da liberação do sedimento, e comportam-se de forma não conservativa, na dependência direta de processos biológicos ou físicos, 
sendo que, em relação à escala de concentração, o silicato foi o mais concentrado seguido pelo nitrato, depois pelo fosfato e por fim pelo $\mathrm{N}$-amoniacal e nitrito.

É importante ressaltar que o conhecimento das condições hidroquímicas dos estuários amazônicos em diferentes períodos sazonais é de suma importância. Os nutrientes dissolvidos, que variaram sazonalmente e longitudinalmente no estuário do rio Paracauari, apresentaram suas concentrações máximas durante o período chuvoso, o qual foi fortemente influenciado pelo La Niña durante esse ano, promovendo carreamento de elevadas concentrações para a baía do Marajó, tornando esse ambiente propicio para o desenvolvimento da biota aquática.

\section{AGRADECIMENTOS}

À CAPES pelo apoio financeiro e aos membros do Grupo de Estudos Marinhos e Costeiros (GEMC-CNPQ) da Universidade Federal do Pará (UFPA).

\section{REFERÊNCIA BIBLIOGRÁFICA}

ANA. Agência Nacional das Águas. Região Hidrográfica Amazônica. Brasil, 2007. Disponível em: http:// www.ana.gov.br/mapa inicial/pgMapaA.asp. Último acesso: 15 mai. 2007.

ALMEIDA, S. S. Ecossistemas de manguezal na Amazônia: Uma abordagem ecológica sobre a presença humana, a flora e a fauna. Boletim do Museu Paraense Emílio Goeldi, Belém, n. 1, p.1-15, 1995.

ALVES, I. C. C. Produtividade primária e hidrologia do sistema flúvio-estuarino do rio Arari (ilha de Marajó- PA). 2010. 113 f. Dissertação (Mestrado em Geologia e Geoquímica) - Universidade Federal do Pará, Belém, 2010.

BARTHEM, B. R. Ocorrência, distribuição e biologia dos peixes da Baía de Marajó, Estuário Amazônico. Boletim do Museu Paraense Emílio Goeldi, Belém, ser. Zool., n.1, p.49-69, 1985.

BAUMGARTEN, M.G.Z., ROCHA, J.M.B., NIENCHESKI, L.F. Manual de análises em Oceanografia Química. Porto Alegre: Ed FURG/Pallotti, 1996. 142 p.

BALLMAN, N., VITORINO, M.I., MOURA, M., DIAS, C., DIAS, J. Oscilação Intrasazonal de Precipitação na Amazônia Oriental durante a La Niña. XII Cong. Brasileiro de Meteorologia, CBME, Gramado, RJ: 5p.

BRADLEY, E.; PHILIP, B. A comparative study of nutrient behavior along the salinity gradient of tropical and temperate estuaries. Estuaries, New Jersey, n.2, p.313-326, 1999.

COHEN, M. C. L., LARA, R. J., SZLAFSZTEIN, C., DITTMAR, T. Mangrove inundation and nutrient dynamics from a GIS perspective. Wetlands Ecology and Management, Germany, n.2, p.81-86, 2004.

CORREL, D.L. Nutrient mass balances for the watershed, headwaters intertidal zone, and basin of the Rhode River Estuary. Limnology and Oceanography, Canmore, n.26, p.1142-1149, 1981.

DEMASTER, D.J., SMITH, W.O.JR., NELSON, D.M., ALLER, J.Y. Biogeochemical processes in Amazon shelf waters: chemical distributions and uptake rate of silicon, carbon and nitrogen. Continental Shelf Research, n.16, p.617-643, 1996.

DHN. DIRETORIA DE HIDROGRAFIA E NAVEGAÇÃO. Tábua das Marés. Brasil, 2008. Disponível em: http:/www.mar.mil.br. Último acesso: 20 set. 2008.

DITTMAR, T.; LARA, R. L., KATTNER, G. River or mangrove? Tracing major organic matter sources in tropical Brazilian coastal waters. Marine Chemistry, v.3, n.4, p.253-271, 2001.

EDMOND, J. M. et al. Biological uptake and accumulation of silica on the Amazon continental shelf. Geochimica et Cosmochimica Acta, n.71, p.1713-1723, 1983.

ELLIOTT, M.; MCLUSKY, D. S. The need for definitions in understanding estuaries. Estuarine, Coastal and Shelf Science, v.55, n.6, p.815-827, 2002.

FERNANDES, G.L. Sub-projeto fitoplâncton. In: LOPES, M.J.S.; FERNANDES, G.L.; MELO, O.T. Estudos do plâncton (fito e zooplâncton) e de fatores físicos e químicos na região estuarina dos rios Anil e Bacanga, São Luís-MA. São Luís: UFPA. FAPEMA, 1994. p. 37-56. 
FRANÇA, C. F.; SOUZA FILHO, P. W. M. Compartimentação morfológica da margem leste da ilha de Marajó: zona costeira dos municípios de Soure e Salvaterra - Estado do Pará. Revista Brasileira de Geomorfologia, n 1, p. 33-42, 2006.

FLORES MONTES, M. J. Variação Nictemeral do fitoplâncton e parâmetros hidrológicos no Canal de Santa Cruz, Itamaracá, PE. 1996. 174 f. Dissertação (Mestrado) - Universidade Federal de Pernambuco, Recife, 1996. GRASSHOFF, K., EHRHARDT, M., KREMLING, K. (Eds.) Methods of Seawater Analysis. 2nd edition. Berlin: Verlag Chemie, 1983. 417 p.

JORDAN, T. E.; CORRELL, D. L.; MIKLAS, J.; WELLER, D. E. Nutrients and chlorophyll at the interface of a watershed and an estuary. Limnology and Oceanography, n.36, p.251-267, 1991.

INMET. Instituto Nacional de Meteorologia. Gráficos das condições registradas. Brasil, 2008. Disponível em: http://www.inmet.gov.br. Último acesso: 15 dez. 2008.

LARA, R. J.; DITTMAN, T. Nutrient dynamics in a mangrove creek (North Brazil) during the dry season. Mangroves and Salt Marshes, n.3, p.185-195, 1999.

LIU, S.M. et al. Factors influencing nutrient dynamics in the eutrophic Jiaozhou Bay, North China. Progress in Oceanography, n.66, p.66-85. 2005.

MAGLIOCCA, A. Some chemical aspects of the marine environment off the Amazon and Pará Rivers, Brazil. Boletim do Instituto Oceanográfico, São Paulo, n.20, p.61-84, 1971.

MCLUSKY, D. S. (Eds.) The estuarine ecosystem. 2. ed., New York: Ed. Chapman e Hal, 1989. 215 p.

MELO, O. T. Comportamento biogeoquímico de nutrientes no estuário do rio Bacanga, Ilha de São Luís - MA. 1998. 115 f. Dissertação (Msc em Geol. e Geouímica) - Universidade Federal do Pará, Belém, 1998. NORIEGA, C. D. et al. Distribuição espacial da biomassa fitoplanctônica e sua relação com os sais nutrientes, no sistema estuarino de Barra das Jangadas (PE - Brasil). Arquivo de Ciências do Mar, n.38, p.5-18, 2005.

PITKÄNEN, H. et al. Late summer trophic conditions in the North-east Gulf of Finland and the River Neva Estuary, Baltic Sea. Estuarine, Coastal and Shelf Science, n.37, p.453-474, 1993.

ROSSETTI, D.F.; GOES, A.M.; VALERIANO, M.M.; MIRANDA, M.C.C. Quaternary tectonics in a passive margin: Marajo Island, northern Brazil. Journal of Quaternary Science, n.22, p.1-15, 2007.

SANTOS, A.; TANCREDI, A.C.N.S.; SILVA, M.S. Limnologia da Amazônia Oriental: caracterização biogeoquímica da parte sul do estuário do rio Amazonas. Acta Limnologica Brasiliensia, n.1, p.113-126, 1986. SANTOS, M. L.; MEDEIROS, C.; MUNIZ, K.; FEITOSA, F.A.N.; SCHWAMBORN, R., MACÊDO, S. Influence of the Amazon and Para Rivers on Water Composition and Phytoplankton Biomass on the Adjacent Shelf. Journal of Coastal Research, v.24, n.3, p.585-593, 2008.

SHAEFFER-NOVELLI, Y. Importância do manguezal e suas comunidades. ALICMAR/Inst. Instituto Oceanográfico USP, São Paulo. 1982. 6 p.

SOUZA, L.S.B.; ROSSETTI, D.F. Análise morfoestrutural na porção leste da Ilha do Marajó/PA. In: SIMPÓSIO BRASILEIRO DE SENSORIAMENTO REMOTO, 14. (SBSR), 2009, Natal. Anais... São José dos Campos: INPE, 2009. p. 3387-3394. DVD, On-line. ISBN 978-85-17-00044-7. (INPE-16052-PRE/10661). Disponível em: <http://urlib.net/dpi.inpe.br/sbsr@80/2008/11.14.09.31>. Último acesso em: 02 jul. 2008. STRICKLAND, J.D.H.; PARSONS, T.R. A practical handbook of seawater analysis. 2. ed. Ottawa: Fisheries Research Board of Canada, 1972. 310 pp.

UNDERWOOD, G.J.C.; KROMKAMP, J. Primary production by phytoplankton and microphytobenthos in estuaries. Advances in Ecological Research. n.29, p.93-153, 1999.

WEBB, R.L. Conceptual models and processes of nutrient cycling in estuaries. In: NEILSON, B.J.; RONIN, L.E. (eds.) Estuaries and Nutrients. New Jersey: Humana Press, 1981. p. 25-46.

WHITE, D.L et al. Spatial and temporal analyses of water quality and phytoplankton biomass in an urbanized versus a relatively pristine salt marsh estuary. Journal of Experimental Marine Biology and Ecology, n.298, p.55-273, 2004.

Trabalho enviado em março de 2015 Trabalho aceito em abril de 2015 